(niobium nitride) is cubic (Fig. 1). This means that the crystallographic symmetry of their devices is broken at the interface between the cubic superconductor and the hexagonal semiconductor. Such broken symmetries can cause unwanted effects at interfaces, and therefore in devices.

This is where the orientation of the superconductor comes into play. Yan et al. grew a layer of the cubic superconductor on a substrate so that its lattice was oriented in a way that makes it look hexagonal. To picture this, imagine looking at a dice at an angle in which the diagonally opposite corners are aligned. What you see is a hexagon, even though the dice is cubic.

The same is true of the cubic superconductor on the substrate: a hexagonal arrangement of atoms is exposed on the surface, and the hexagonal semiconductor (aluminium nitride) aligns with this when it forms on top of the superconductor. As a result, the aluminium nitride is not perturbed by broken crystallographic symmetry at the interface, and forms an undistorted layer, as needed for the growth of an HEMT structure. Indeed, the authors observed the formation of certain quantum oscillations in their device; the presence of these oscillations is considered a benchmark of high crystal quality.

Yan et al. went on to measure the currentvoltage profile of their superconductor-HEMT structure. They observed that this profile of the HEMT is modified by a superconductor-tometal transition in niobium nitride, and generates a negative differential resistance (NDR) - a property that can be used to increase the power of electrical signals. NDR devices have been known since the end of the nineteenth century $^{8}$ and include the Gunn diode ${ }^{9}$, which is widely used to generate microwaves in sensors and measuring instruments. Such devices are of great value for electronic systems that use high-frequency, high-power signals - exactly what is needed in telecommunications networks. In Yan and colleagues' device, NDR can be switched on or off simply by making the temperature lower or higher than the critical temperature for superconductivity (the temperature below which superconductivity occurs).

Combining materials that have different electronic properties without breaking the crystallographic symmetry at the interface is a remarkable feat. However, the mobility of electrons in the device is currently rather low; much higher mobilities can be achieved in devices that use indium arsenide. Achieving mobilities comparable to those of indium arsenide will be extremely challenging. Moreover, the separation between the superconductor and the $2 \mathrm{D}$ electron gas - free electrons that are confined to move in only two dimensions generated in the device will need to be reduced to enable promising quantum effects.

A future goal could be to use the authors' system to generate and observe Majorana fermions ${ }^{10}$ - a type of quasiparticle that would be useful for quantum computing - at the superconductor-semiconductor interface ${ }^{11}$. Charge carriers in electronic devices can be scattered (for example, by crystal defects), and the average time between scattering events needs to be long to stabilize these quasiparticles. Yan et al. calculate that the charge-carrier scattering time in their devices is impressively long (66 femtoseconds; $1 \mathrm{fs}$ is $10^{-15} \mathrm{~s}$ ), but the scattering times will need to be at least 100 times longer, similar to the scattering time in indium arsenide ${ }^{12}$, to stabilize Majorana fermions. It remains to be seen whether this can be achieved in the authors' devices.

Ultimately, Yan and colleagues' work will inspire and accelerate efforts to grow nitride superconductors and nitride semiconductors that enable the ultra-high operating efficiency, structural perfection and opportunities for manipulating electronic properties that have already been achieved in interfaces involving indium arsenide. Because, at the end of the day, the interface is the device.
Yoshiharu Krockenberger and Yoshitaka Taniyasu are in the Materials Science Laboratory, NTT Basic Research Laboratories, Atsugi, Kanagawa 243-0198, Japan. e-mails:yoshiharu.k@lab.ntt.co.jp; taniyasu.yoshitaka@lab.ntt.co.jp

1. Yan, R. et al. Nature 555, 183-189 (2018).

2. Kroemer, H. Quasi-Electric Fields and Band Offsets: Teaching Electrons New Tricks (Nobel Lecture, 8 Dec 2000).

3. Tsujimoto, M. et al. Phys. Rev. Lett. 108, 107006 (2012).

4. Kjaergaard, M. et al. Phys. Rev. Appl. 7, 034029 (2017).

5. Senapati, K., Blamire, M. G. \& Barber, Z. H. Nature Mater. 10, 849-852 (2011).

6. Akasaki, I. Fascinated Journeys into Blue Light (Nobel Lecture, 8 Dec 2014).

7. Mimura, T. IEEE Trans. Microw. Theory Technique 50, 780-782 (2002).

8. Frith, J. \& Rodgers, C. Lond. Edinb. Dubl. Phil. Mag J. Sci. 42, 407-423 (1896)

9. Gunn, J. B. Solid State Commun. 1, 88-91 (1963)

10. Nichele, F. et al. Phys. Rev. Lett. 119, 136803 (2017).

11. Krogstrup, P. et al. Nature Mater. 14, 400-406 (2015).

12.Shojaei, B. et al. Phys. Rev. B 94, 245306 (2016).

\title{
EVOLUTION
}

\section{Mountains of diversity}

\begin{abstract}
A large-scale analysis of bird diversity and evolution on mountains around the globe explores the relationships between elevation, species richness and the rate of formation of new species. SEE LETTER P. 246
\end{abstract}

\section{ALEXANDER ZIZKA \& ALEXANDRE ANTONELLI}

$\mathrm{M}$ ountain chains are global centres of biological diversity - they harbour one-third of all terrestrial species ${ }^{1}$. These places have long fascinated biologists ${ }^{2}$, but are notoriously difficult to explore and study. Our knowledge of the distribution of species diversity on mountains is incomplete, as is our understanding of how species richness (the total number of species) and the rates of formation of new species (speciation) vary in single mountain ranges. On page 246, Quintero and Jetz ${ }^{3}$ tackle these issues by studying the diversity and evolution of birds on the 46 major mountain ranges of the world.

Mountains can differ substantially in the environment they provide, depending on factors such as bedrock, ruggedness, climatic conditions and the amount of energy available in the region. Moreover, mountains are often far apart, and organisms inhabiting such places can persist in genetically isolated populations owing to factors including terrain complexity and the high variation of habitat types along elevational gradients. Isolated populations often adapt to the local environmental and ecological conditions. When such populations are no longer capable of reproducing with one another, they form new species ${ }^{4}$. One example of this is the hummingbird Aglaiocercus kingii, which is found only in the Andes of South America (Fig. 1).

Quintero and Jetz used large-scale data sets of current distributions of bird species, mined from existing databases and publications, to characterize the relationship between elevation above sea level and species richness. The authors amalgamated data for 9,993 species, representing essentially all the birds that are currently known. Although the patterns observed in different regions vary, the overall trend for most regions is a hump-shaped curve in which species richness is highest at middle elevations, and decreases as elevation increases.

The result confirms findings from previous studies of plants and birds $s^{5,6}$. This type of pattern might be driven by the smaller area available for speciation at higher elevations and because the environmental conditions there are more extreme than those on lowlands. For example, large temperature fluctuations between day and night, and an increased exposure to radiation and wind at higher elevations could limit the number of species that can cope with such conditions.

The authors used some innovative approaches for their data analysis. They aimed 


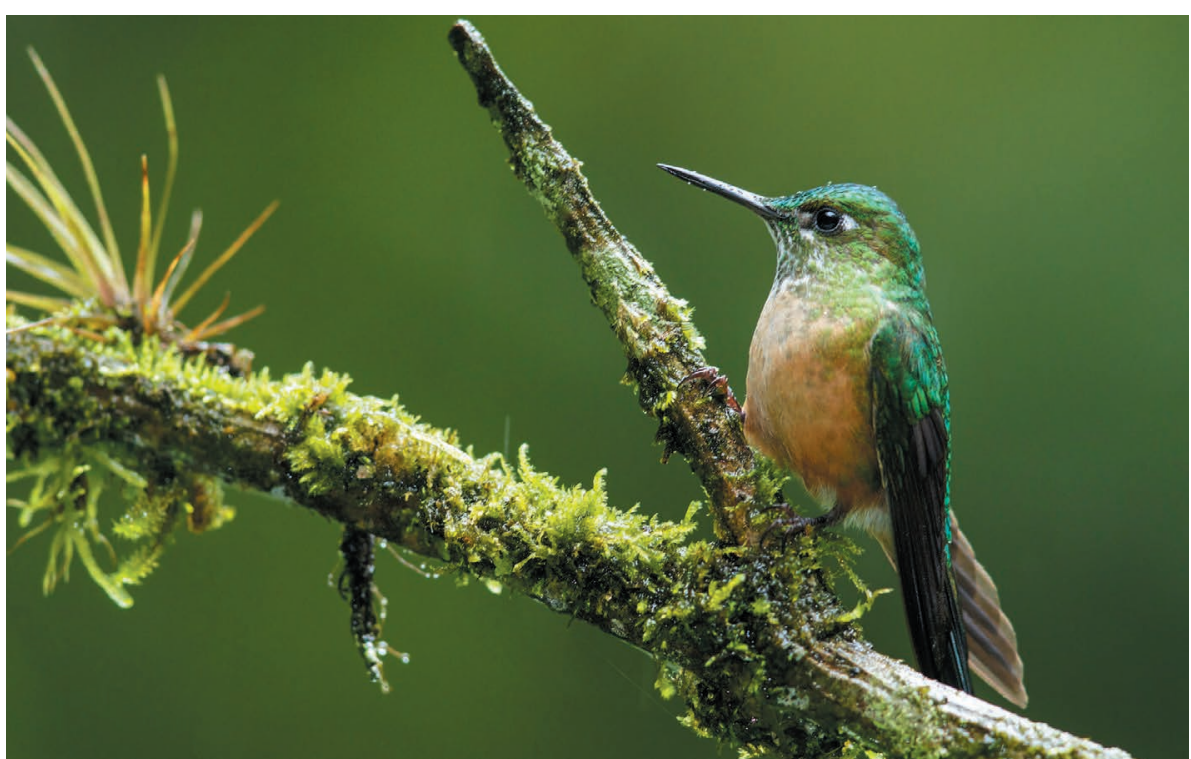

Figure 1 | The hummingbird Aglaiocercus kingii in Ecuador. This species is confined to the Andes mountains of South America. Quintero and Jetz ${ }^{3}$ have developed an approach for studying bird distributions on mountains around the world that might help to address how and when biological diversity evolved along elevational gradients.

to capture the 3D structure of biodiversity data by combining elevation and species information. They performed their analyses by grouping the bird data into 'sliced sections' corresponding to trapezoidal prisms that encompass a particular elevation range. This allowed them to assess mountain complexity in a way that improves on conventional ecological methods that often neglect elevation.

Some biodiversity analyses can be affected by issues such as the mid-domain effect, in which a species-richness peak occurs around the centre of a region because of the spatial overlap of species' ranges ${ }^{7}$. The authors developed a subsampling approach that offers a way to address this issue. They counted species, but also factored in the total area that each species occupies when determining species' contributions to species richness. This method also uses a complex randomization procedure that takes a modelling approach to estimate the species' metrics.

Their analysis using this subsampling approach revealed the surprising result that there is a linear decrease in species richness as elevation increases. Nevertheless, one concern is that the subsampled species-richness estimates from this method may not be directly comparable with estimates of species richness calculated in the conventional way - as the total number of species. In addition, the size of each species' range might be a factor linked to its evolutionary history, and therefore relevant for understanding the evolution of mountain species. Additional research might be needed to assess the applicability of this subsampled diversity metric.

Another, perhaps even more interesting finding made by Quintero and Jetz concerns the process underlying the observed species diversity patterns. The authors used previously estimated ${ }^{8}$ information on the evolutionary relationships between the bird species that they studied to calculate the rate of speciation along elevational gradients. They found that this rate is inversely related to subsampled species richness: that is, species are formed at the highest rates where the species richness is lowest, which corresponds to mountaintops. The authors' explanation for this is that environmentally stable lowlands have high diversity, whereas at higher elevations, diversity is governed mainly by frequent immigrations and rapid species replacement during periods of climate change.

A major limitation for studies of biological diversity on mountains is the scarcity of available data. Quintero and Jetz's study uses existing diversity data that have a resolution of at least 110 kilometres horizontally and 500 metres in elevation. This kind of scale can be rather coarse for many mountains, given that environmental and ecological conditions can vary considerably over distances of just a few hundred metres. Although birds are the best geographically documented group of organisms on Earth, with more than 564 million publicly available records (see www.gbif.org), it might come as a surprise that their diversity in many mountains remains poorly documented.

Unfortunately, the geological data of most relevance to biologists are lacking. Quintero and Jetz therefore had to simplify geological complexity in their analyses by using averaged values for key variables, such as the age of mountains. These factors, together with ecological interactions between species, might influence the speciation process ${ }^{9}$, and can vary in a single mountain range.

Speciation rates are also difficult to estimate, especially over long timescales and for groups, such as birds, that lack a rich fossil record. One potential drawback of the new study is that many relationships between species, and their estimated time of origin, have been calculated on the basis of limited genetic information and with methods that do not take into account the difficulties that sometimes arise during the generation of phylogenetic trees. In some cases, proposed relationships might rely only on comparisons of bird shape and form (morphology) rather than on genetic data.

There is still a long way to go before the phylogeny of birds is fully understood ${ }^{10}$. Largescale initiatives are under way to sequence the genomes of all bird species as a way to determine more-reliable estimates of the relationships between birds and to improve understanding of their evolutionary history ${ }^{11}$.

Quintero and Jetz's results reveal general and unexpected relationships between elevation, species richness and diversification. Additional data collection in the field by scientists and birdwatchers will be essential and, along with data integration and analysis of the sort spearheaded by Quintero and Jetz, should provide additional insights. It will be particularly interesting to see whether the trends reported by Quintero and Jetz hold true for the rest of the world's species, the diversity and distribution of which are poorly known even at the global level $^{12}$ - let alone along elevational gradients on mountains.

Alexander Zizka and Alexandre Antonelli are at the Gothenburg Global Biodiversity Centre, SE-405 30 Gothenburg, Sweden, and in the Department of Biological and Environmental Sciences, University of Gothenburg. A.A. is also at the Gothenburg Botanical Garden and in the Department of Organismic and Evolutionary Biology, Harvard University, Cambridge,

Massachusetts.

e-mail:alexandre.antonelli@bioenv.gu.se

1. Spehn, E. M., Rudmann-Maurer, K. \& Körner, C. Plant Ecol. Divers. 4, 301-302 (2011)

2. von Humboldt, A. \& Bonpland, A. Essai sur la Géographie des Plantes (Schoell, Cotta, 1807)

3. Quintero, I. \& Jetz, W. Nature 555, 246-250 (2018)

4. Hoorn, C., Mosbrugger, V., Mulch, A. \& Antonelli, A. Nature Geosci. 6, 154 (2013)

5. Kessler, M., Herzog, S. K., Fjeldså, J. \& Bach, K. Divers. Distrib. 7, 61-77 (2001).

6. McCain, C. M. Glob. Ecol. Biogeogr. 18, 346-360 (2009).

7. Colwell, R. K. \& Lees, D. C. Trends Ecol. Evol. 15, 70-76 (2000)

8. Jetz, W., Thomas, G. H., Joy, J. B., Hartmann, K. \& Mooers, A. O. Nature 491, 444-448 (2012)

9. Condamine, F. L., Antonelli, A., Lagomarsino, L. P., Hoorn, C. \& Liow, L. in Mountains, Climate, and Biodiversity (eds Hoorn, C., Perrigo, A. \& Antonelli, A.) (Wiley, in the press).

10.Ricklefs, R. E. \& Pagel, M. Nature 491, 336-337 (2012).

11.Zhang, G., Jarvis, E. D. \& Gilbert, M. T. P. Science 346, 1308-1309 (2014).

12.Mora, C., Tittensor, D. P., Adl, S., Simpson, A. G. B. \& Worm, B. PLoS Biol. 9, e1001127 (2011).

This article was published online on 21 February 2018. 